\title{
Polarization Control via He-ion Beam Induced Nanofabrication in Layered Ferroelectric Semiconductors
}

\author{
A. Belianinov, ${ }^{1,2}$ V. Iberi, ${ }^{2,4}$ A. Tselev, ${ }^{1,2,3}$ M. Susner, ${ }^{5}$ M. McGuire, ${ }^{5}$ D. Joy, ${ }^{2,4}$ S. Jesse, ${ }^{1,2}$ A. \\ J. Rondinone, ${ }^{2}$ S. V. Kalinin, ${ }^{1,2}$ O. S. Ovchinnikova ${ }^{1,2 *}$ \\ 1. The Institute for Functional Imaging of Materials and the Center for Nanophase Materials \\ Sciences, Oak Ridge National Laboratory, Oak Ridge, TN 37831 \\ 2. Center for Nanophase Materials Sciences, Oak Ridge National Laboratory, Oak Ridge, TN \\ 37831 \\ 3. Department of Physics and Astronomy, University of Tennessee, Knoxville, Knoxville TN \\ 37996 \\ 4. Department of Materials Science and Engineering, University of Tennessee, Knoxville, \\ Knoxville TN 37996 \\ 5. Materials Sciences and Technology Division, Oak Ridge National Laboratory, Oak Ridge, TN \\ 37831
}

Rapid advancements in nanoscience rely on improvements in manipulating matter at finer length scales. Currently, well understood, robust resist-based lithography, carries the brunt of nanofabrication, however local electron, ion and physical probe methods are improving as well. These developments are driven by the ability to fabricate without the multi-step preparation processes that can result in sample contamination from the resists and solvents. Furthermore probe based methods extend beyond nanofabrication to nanomanipulation and imaging, vital ingredients to rapid transition to testing and manufacturing of layered 2D heterostructured devices.

In this work we demonstrate that helium ion interaction in a Helium Ion Microscope (HIM) with the surface of bulk copper indium thiophosphate (CITP) $\mathrm{CuM}_{\mathrm{III}} \mathrm{P}_{2} \mathrm{X}_{6}(\mathrm{M}=\mathrm{Cr}$, In; $\mathrm{X}=\mathrm{S}, \mathrm{Se})$ [1] result in the controlled loss of ferrielectric domains, and growth of cylindrical nanostructures with enhanced conductivity, with material volumes scaling to the dosage of the beam. Figure 1 summarizes some of our finding by illustrating the sample morphology as seen by an optical microscopy (Fig. 1(a,b)), the types of structures forming on the surface as a function in helium ion beam dose (Fig. 1(c)), and the physical parameters as a function of the beam dose. These structures are air and temperature stable up to $100{ }^{\circ} \mathrm{C}$. The nanostructures are oxygen rich, sulfur poor, with the copper concentration virtually unchanged as confirmed by Energy Dispersive Xray (EDX). Quantitative chemical identification was performed by Secondary Ion Mass Spectrometry (SIMS). Scanning Electron Microscopy (SEM) image contrast as well as Scanning Microwave Microscopy (SMM) measurements suggest enhanced conductivity of the formed particle, whereas Atomic Force Microscopy (AFM) based measurements indicate that the resulting structures have lower dissipation and a lower Young's modulus. [2] 

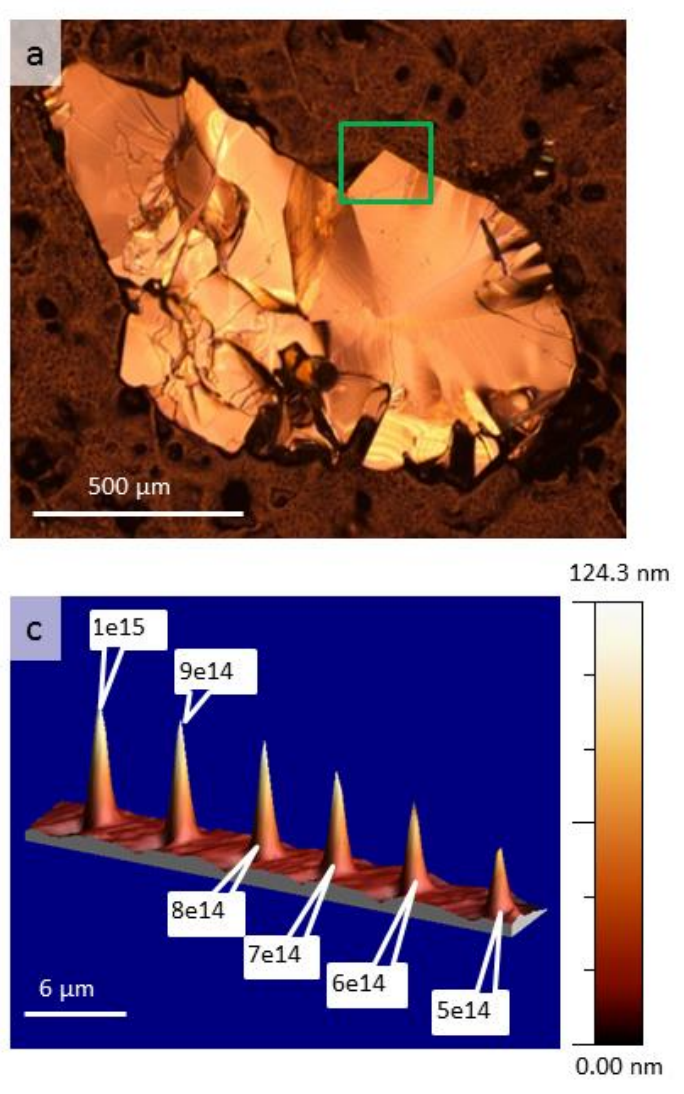

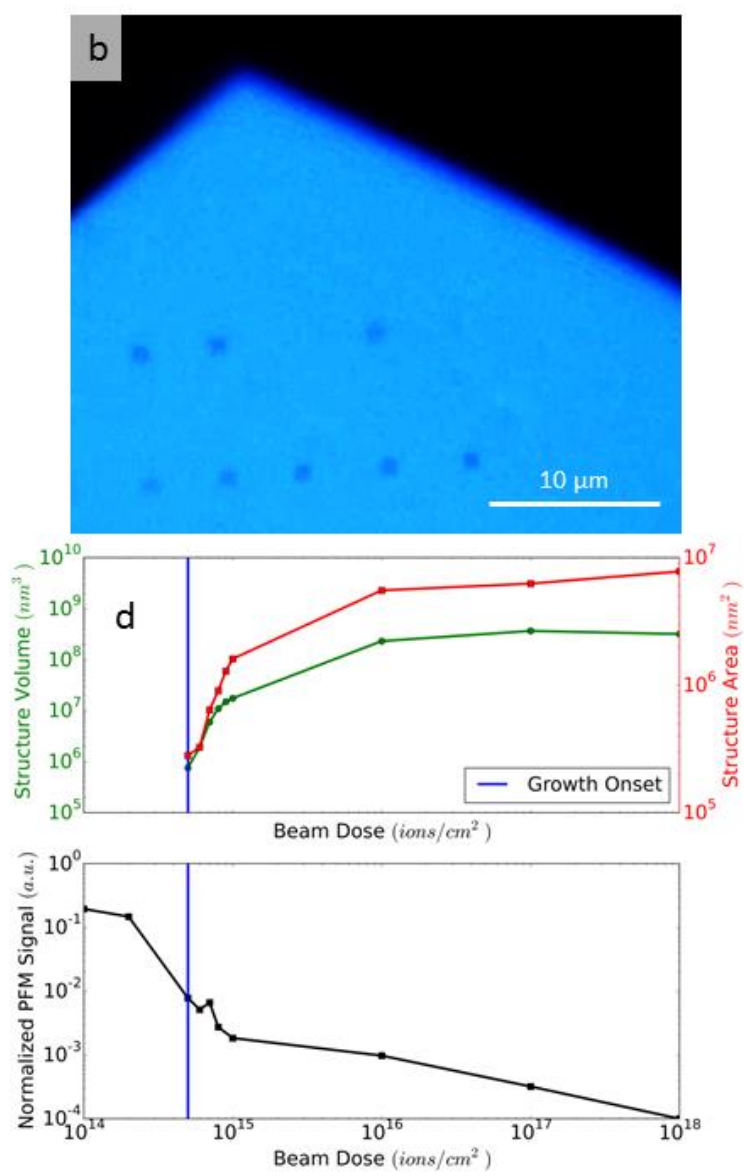

Figure 1. Sample morphology and He ion induced growth (a) Optical image of exfoliated $\mathrm{CuInP}_{2} \mathrm{~S}_{6}$ crystal prior to exposure to the helium-ion beam. (b) Optical image of boxed area, highlighted by the green box, in panel (a) showing visible surface transformation caused by exposure to the helium-ion beam under varying ion beam dosages. (c) AFM topography image showing pillar growth on the $\mathrm{CuInP}_{2} \mathrm{~S}_{6}$ crystal surface under exposure of varying ion beam dosages. (d) Plot of structure volume, area and the average normalized PFM signal as a function of ion beam dose across a $1 \mathrm{um}^{2}$ area square with a $0.5 \mu$ s exposure time measure using AFM topography.

\section{References}

[1] A Belianinov, Q He, A Dziaugys, P Maksymovych, E Eliseev, A Borisevich, A Morozovska, J Banys, Y Vysochanskii, SV Kalinin, CuInP2S6 Room Temperature Layered Ferroelectric, Nano Letters, 15(6), 3808-3814, 2015

[2] Research was supported) and partially conducted (AFM, HIM) at the Center for Nanophase Materials Sciences, which is sponsored at Oak Ridge National Laboratory by the Scientific User Facilities Division, Office of Basic Energy Sciences, US Department of Energy. This work was also supported (M. S., M. M.) and partially conducted (material growth) by the U.S. Department of Energy, Basic Energy Sciences, Materials Sciences and Engineering Division. 